\title{
Measurement of a three-dimensional photonic band gap in a crystal structure made of dielectric rods
}

\author{
E. Özbay \\ Ames Laboratory and Microelectronics Research Center, Iowa State University, Ames, Iowa 50011
}

A. Abeyta

Ames Laboratory and Department of Physics and Astronomy, Iowa State University, Ames, Iowa 50011

G. Tuttle

Department of Electrical Engineering and Microelectronics Reseach Center, Iowa State University, Ames, Iowa 50011

M. Tringides

Ames Laboratory and Department of Physics and Astronomy, Iowa State University, Ames, Iowa 50011

R. Biswas

Ames Laboratory and Microelectronics Research Center, Iowa State University, Ames, Iowa 50011

C. T. Chan, C. M. Soukoulis, and K. M. Ho

Ames Laboratory and Department of Physics and Astronomy, Iowa State University, Ames, Iowa 50011

(Received 14 April 1994)

\begin{abstract}
We have designed, fabricated, and tested a three-dimensional photonic-band-gap structure. The structure is built by an orderly stacking of dielectric rods. A network analyzer is used to measure the transmission and dispersion relations of electromagnetic waves along several crystal directions. The experimental results are in good agreement with the theoretical calculations. The structure is different from that previously suggested, as it is already used to improve the midgap frequency by almost two orders of magnitude, and it offers a viable geometry to extend the photonic band gap to optical regimes.
\end{abstract}

The existence in three-dimensional periodic structures of a photonic band gap, or a frequency region where the propagation of electromagnetic (EM) waves is forbidden for all wave vectors, has several potential scientific applications. ${ }^{1}$ These applications include zero-threshold semiconductor lasers, the absence of spontaneous emission, single-mode light-emitting diodes, novel resonators and cavities, and efficient microwave antennas. ${ }^{2-4}$ To realize most of these applications it is crucial to have a photonic-band-gap crystal lattice that can be easily and reproducibly fabricated. The theoretical calculations of Ho, Chan, and Soukoulis predicted that periodic dielectric materials with a diamondlike symmetry would have a full three-dimensional photonic band gap. ${ }^{5,6}$ One structure was fabricated in the millimeter length scale by drilling three sets of cylinders in a dielectric material at $35.26^{\circ}$ off normal, and was confirmed to have a full three-dimensional photonic band gap in the microwave regime between $13-16 \mathrm{GHz}^{7}$ While demonstrating the existence of the photonic gap, the fabrication of such a three-cylinder structure is a very sophisticated process that cannot be easily reproduced or extended to optical wavelengths. In this paper we present measurements on a three-dimensional dielectric lattice that can be easily fabricated, and possesses a full photonic band gap in the microwave regime $(10-20 \mathrm{GHz})$. We believe this is a viable structure for producing photonic crystals in the millimeter wave and optical regime.

We note that two-dimensional triangular lattices of dielectric rods or of air columns in a dielectric material have been fabricated, which reveal two-dimensional photonic band gaps agreeing well with photonic-bandstructure calculations. ${ }^{8-10}$ However, EM waves can propagate freely in the direction parallel to the rods. Moreover, predictions of small photonic band gaps $(\approx 3 \%)$ exist in simple cubic dielectric structures ${ }^{11}$ that also may be hard to realize experimentally. There is an urgent need to fabricate a simple crystal structure with a sizable photonic band gap, and this is described in this paper.

The photonic-band-gap crystal we have designed and fabricated consists of simple one-dimensional dielectric rods as the basic building blocks. The photonic crystal is assembled by stacking together layers of dielectric rods, with each layer consisting of parallel rods with a centerto-center separation of $a$. The rods are rotated by $90^{\circ}$ in each successive layer. Starting at any reference layer, the rods of every second neighboring layer are parallel to the reference layer, but shifted by a distance of $0.5 a$ perpendicular to the rod axes. This results in a stacking sequence that repeats every four layers. This lattice has face-centered-tetragonal (fct) lattice symmetry with a basis of two rods. The photonic band gap is not sensitive to the cross-section shape of the rods. This structure has a photonic band gap when both the filling ratio and the dielectric contrast meet certain requirements. ${ }^{12}$

In the microwave frequency regime, the photonic crystal can be built using commercially available cylindrical 
alumina rods with a refractive index of 3.1. The alumina rods were $0.318 \pm 0.013 \mathrm{~cm}$ in diameter and $15.24 \mathrm{~cm}$ long, and a center-to-center separation of $1.123 \mathrm{~cm}$ was chosen to obtain a dielectric filling ratio of $\sim 0.26$. Once the first layer has been built, the second layer is glued on top of the first layer by putting a drop of glue at each point where the rods touch. We used a special glue that has a similar dielectric constant as alumina, and our theoretical analysis has shown that the small amount of glue ( $<5 \%$ of the rod volume) used in this structure does not affect the overall photonic-band-gap properties as the structure without the glue. The third layer is stacked with the rods shifted by $a / 2=0.5615 \mathrm{~cm}$ with respect to the first layer, and the structure is simply built by successive stacking of layers of rods. Figure 1 shows a picture of a stacked layer that has four conventional unit cells in the $z$ direction (16 stacked layers), and 13 or 14 rods in each layer. We define the plane of each rod layer as the $x-y$ plane with the rod axes defining the (110) directions in this plane. The symmetry of this crystal is such that the electromagnetic wave propagation along the $z$ axis is degenerate for both polarizations. However, for propagation along the rods (100 direction), the propagation for the polarization vector $\mathrm{e}$ in the $x-y$ plane is nondegenerate with $e$ along the $z$ axis. Figure 2 shows the corresponding first Brillouin zone (BZ) of the photonic crystal which has an fct symmetry.

We have measured the transmission and phase dispersion proprieties of the structure using a Hewlett-Packard 8510A network analyzer. Standard-gain horn antennas are used to transmit and receive the EM radiation. Surroundings of the test setup are covered with absorbers to build an anechoic chamber resulting in a sensitivity of 70 dB. The setup is calibrated for phase and power measurements of the EM wave transmitted between the antennas. The structure is placed in the beam path of the EM wave transmitted from the source antenna. Additional absorbers are used to surround the side faces of the structure to minimize the leakage due to surface states.

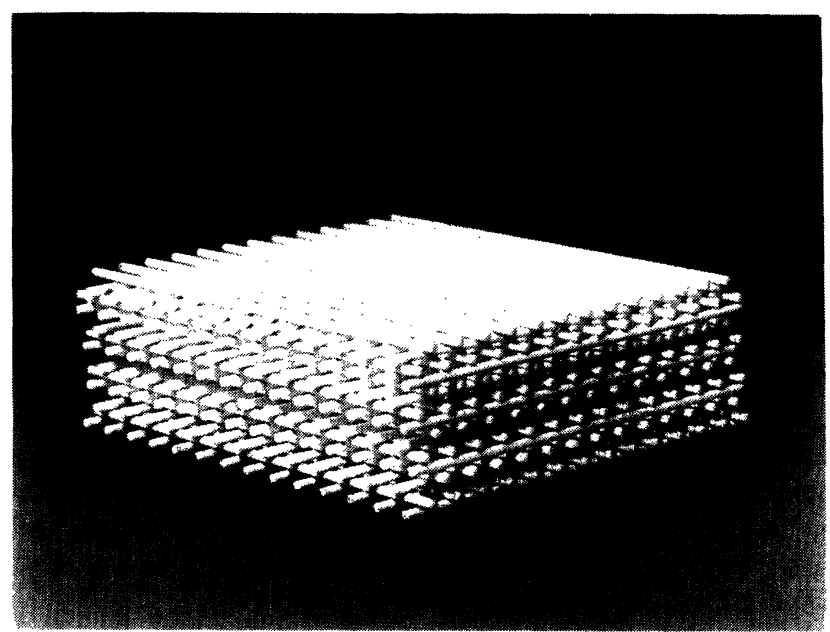

FIG. 1. Photograph of the three-dimensional photonic-gap structure. The crystal is built by stacking 16 layers of alumina rods (13 or 14 alumina rods on each layer) corresponding to four unit cells in the stacking direction.

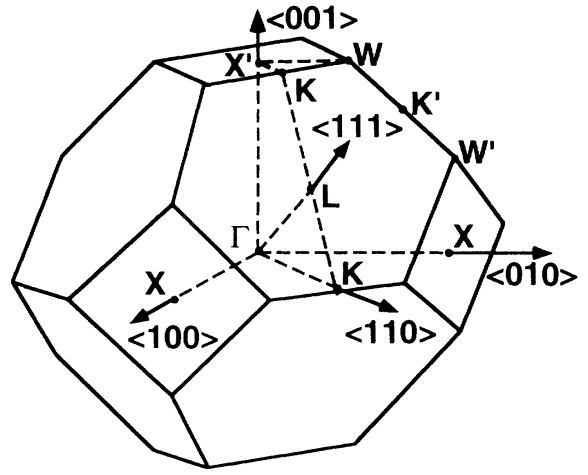

FIG. 2. First Brillouin zone of the photonic-gap structure which carries an fet symmetry. $\langle 001\rangle$ direction corresponds to propagation along the stacking direction. $\langle 011\rangle$ direction corresponds to propagation along the rod axes.

Two separate pairs of antennas are used to cover the $6-20-\mathrm{GHz}$ measurement range. A pair of monopole antennas are used to obtain the phase dispersion at lower frequencies $(1-6 \mathrm{GHz})$. Propagation characteristics along the $z$ axis are obtained by facing the top surface $(x-y$ plane) of the structure to the source antenna. Propagation along the (110) direction is obtained by facing the side surfaces of the structure to the source antenna. Different polarizations are achieved by rotating the antennas $90^{\circ}$. In order to map out different regions of the first $\mathrm{BZ}$, the incidence angle is changed by rotating the photonic-gap crystal.

Transmission measurements are performed to determine the photonic gaps of the crystal for propagation along major axes. Figure 3(a) shows the transmission measurement for propagation along the rods with the polarization vector $e$ along the $z$ axis. The lower edge of the photonic gap is at $11.9 \mathrm{GHz}$, while the upper edge is at $14.0 \mathrm{GHz}$. This is very close to the calculated band-gap edges of 11.7 and $13.7 \mathrm{GHz}$. The average attenuation within the gap is around $35 \mathrm{~dB}$, which is mostly due to the leakage of the EM power from the surface states resulting from the finite size of the crystal. The valenceband edge at this direction and polarization corresponds to the maximum of the valence band in all directions, while the conduction-band edge is slightly higher than the calculated minimum conduction band $(13.3 \mathrm{GHz})$, which occurs at another propagation direction corresponding to the $L$ point on the BZ. ${ }^{12}$ Figure $3(\mathrm{~b})$ shows the transmission characteristics of the propagation along the $z$ axis for a structure with four unit cells along the $z$ axis. The lower edge of the gap starts at $10.7 \mathrm{GHz}$, while the upper edge is around $17.4 \mathrm{GHz}$. The calculated band-gap edges for this propagation are 11.1 and 16.8 $\mathrm{GHz}$. The conduction-band edge for this propagation direction is not well defined and the amount of power transmitted at conduction-band frequencies is $10 \mathrm{~dB}$ less than the power transmitted at valence-band frequencies, mostly due to reflection from the top surface of the structure. The maximum attenuation within the band gap is measured to be more than $70 \mathrm{~dB}$, and the measurement is 
limited by the sensitivity of the setup. We have determined that the average attenuation per unit cell for propagation along the $z$ axis is approximately $17 \mathrm{~dB}$. This is especially important for future applications, as three unit cells (for a total of 12 stacked layers) will result in a band gap with 50-dB attenuation, which is large enough for most applications.

The phase measurement in our previously described experimental setup gives the net phase difference $(\phi)$ between the phase of the EM wave propagating through the photonic crystal and the phase of the EM wave propagating in free space, for a total crystal thickness of $L$. This can be used to measure the wave vector $k$ of the crystal at each frequency by

$$
\begin{aligned}
\phi & =k L-2 \pi \frac{f}{c} L, \\
k & =\frac{\phi}{L}+2 \pi \frac{f}{c},
\end{aligned}
$$

where $f$ is the frequency of the EM wave, and $c$ is the velocity of light.

The mapping of the BZ surface is performed by holding the crystal at an incidence angle $\theta$ with the electromagnetic wave, and measuring the photonic gap. From Snell's law, it is known that the parallel component of the wave vector of the incoming wave, $k_{\|}$, will be con-
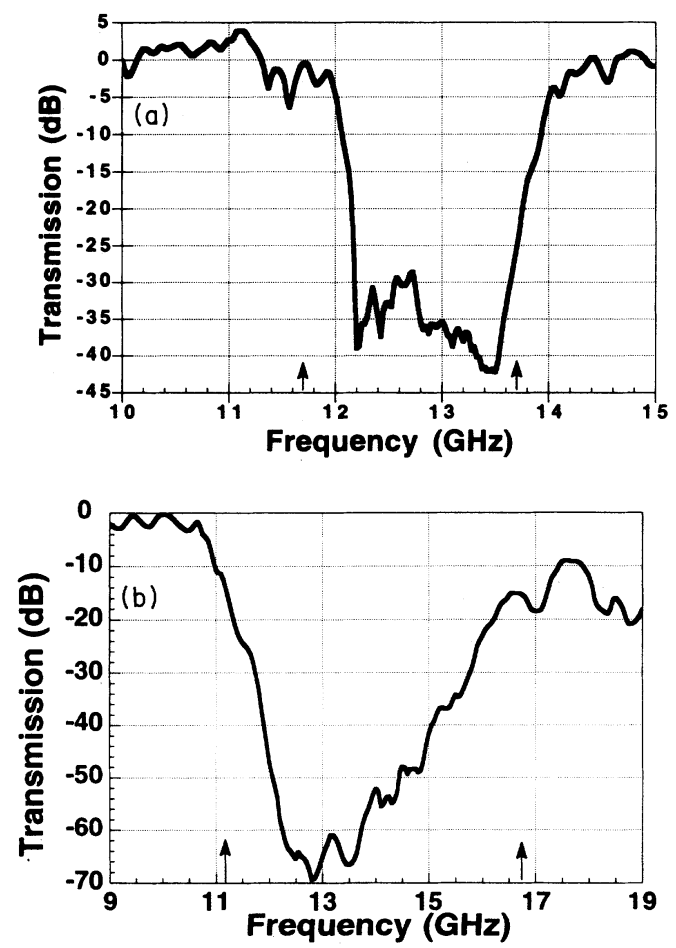

FIG. 3. (a) Transmission characteristics of the photonic-gap structure along the $x$ axis when an $E$ field is perpendicular to the rods. (b) Transmission characteristics along the stacking direction ( $z$ axis). There is no $E$-field polarization dependence for this propagation direction. The arrows show the calculated positions of band edges in both plots. served in the crystal:

$$
k_{\|}=2 \pi \frac{f}{c} \sin \theta \text {. }
$$

Using the energy-band diagram of the photonic crystal on the Brillouin zone surface, we express the frequency as a function of $k_{\|}$in Eq. (3), resulting in a closed equation, which is solved by numerical means to find the corresponding $k_{\|}$for each incidence angle. Once $k_{\|}$is known, the total wave vector of the crystal for each incidence angle is calculated. The measured photonic gap for each incidence angle is then compared with the theoretical photonic gap. For certain parts of the $\mathrm{BZ}$ where $k_{\|}$of the photonic crystal is too large, we used dielectric prisms to increase the parallel wave-vector component of the incoming electromagnetic wave.

These two techniques, phase and angle measurements, have been used to map out the energy-band diagrams of the photonic-gap structure. Figures $4(a)$ and $4(b)$ compare the experimental and theoretical energy-band diagrams along major points of the BZ. The circles represent the measurements performed with polarization vector $\mathrm{e}$ in the $x-y$ plane of the crystal, while the squares represent the measurements performed with e along the $z$ axis. Figure $4(a)$ shows the dispersion relation along $\Gamma-K-L-K-X^{\prime}-\Gamma$, which has strong polarization depen-
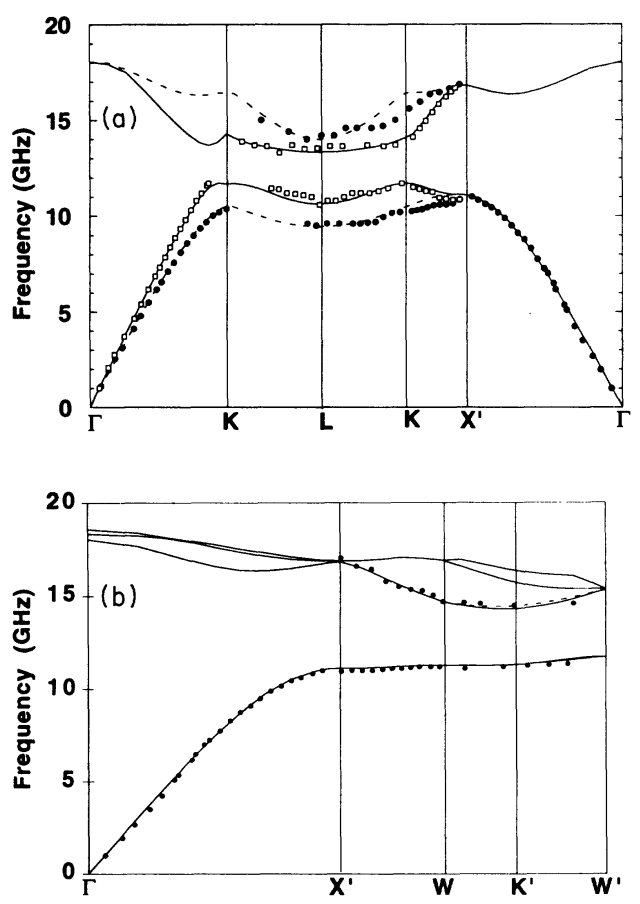

FIG. 4. (a) Frequency vs wave-vector dispersion along $\Gamma-K-L-K-X^{\prime}-\Gamma$ points in the BZ. The dashed and solid curves represent the calculations for $E$-field polarization parallel and perpendicular to the rod axes. The circles represent the measurements performed with an $E$ field parallel to the rod axes polarization, while the squares represent the measurements performed with an $E$ field perpendicular to the rod axes polarization. (b) Frequency vs wave-vector dispersion along $\Gamma-X^{\prime}-W-K^{\prime}-W^{\prime}$ points in the BZ. 
dence. Figure 4(b) shows the dispersion relation along $\Gamma-X^{\prime}-W-K^{\prime}-W^{\prime}$, where the conduction band shows a slight polarization dependence, while the valence band is doubly degenerate. The frequency region between the valence and conduction bands corresponds to the forbidden photonic band gap. The experimental gap frequencies are in excellent agreement with the calculated gap values.

As mentioned earlier, the maximum of the valence band occurs at the $K$ point, while the minimum of the conduction band occurs at the $L$ point. This gives a calculated ratio of band-gap to mid-gap frequency of $13 \%$, while the measured ratio of band-gap to mid-gap frequency is $12 \%$. Theoretical calculations predict the existence of a gap for refractive-index contrasts as low as 1.9, which makes the structure viable of a wide range of dielectric materials. ${ }^{12}$

The structure described here offers unique advantages over previously suggested photonic-gap structures. ${ }^{8,10}$ It exhibits a three-dimensional photonic gap while using nonoverlapping dielectric rods. The resulting photonic gap is really robust and is insensitive to small amounts of disorder. It can also be easily built at millimeter and submillimeter wave frequencies. Using micromachined silicon wafers, we have already fabricated and tested photonic-gap crystals with $100-$ and $500-\mathrm{GHz}$ gap frequencies. $^{13,14}$ These results correspond to the highest mid-gap frequency among all reported photonic-gap structures with full photonic band gaps. The structure also offers the possibility of extending this stacking technique to the optical regime using nanolithography techniques. Although this is not an easy task, the only alternate method is the three-angle etching technique, and structures built around this method will be limited to a few number of layers due to expansion of etch beam. So, our three-dimensional photonic-gap structure has several advantages, and it brings a viable solution to the construction of three-dimensional small-scale photonic-gap structures.

In summary, we have built and tested a threedimensional photonic-band-gap structure. The transmission properties and the energy-band structures of the photonic-gap crystal are measured by using a microwave network analyzer. The photonic crystal exhibits a full three-dimensional band gap between 11.7 and $13.5 \mathrm{GHz}$. The experimental results are in good agreement with the theoretical predictions along several crystal directions. The structure is different from previously suggested photonic-gap structures, as it offers a viable design that can be built at smaller scales to achieve photonic-gap materials at millimeter wave and optical frequencies.

Ames Laboratory is operated by the U.S. Department of Energy by Iowa State University under Contract No. W-7405-Eng-82. This work is supported by the Director for Energy Research, Office of Basic Energy Sciences and Advanced Energy Projects, and also partially supported by a State of Iowa appropriation.
${ }^{1}$ E. Yablonovitch and T. J. Gmitter, Phys. Rev. Lett. 63, 1950 (1989).

${ }^{2}$ E. Yablonovitch, J. Opt. Soc. Am. B 10, 283 (1993).

${ }^{3}$ D. L. Bullock, C. C. Shih, and R. S. Margulies, J. Opt. Soc. Am. B 10, 399 (1993).

${ }^{4}$ E. R. Brown, C. D. Parker, and E. Yablonovitch, J. Opt. Soc. Am. B 10, 404 (1993).

${ }^{5}$ K. M. Ho, C. T. Chan, and C. M. Soukoulis, Phys. Rev. Lett. 65, 3152 (1990).

${ }^{6}$ C. T. Chan, K. M. Ho, and C. M. Soukoulis, Europhys. Lett. 16, 563 (1991).

${ }^{7}$ E. Yablonovitch, T. J. Gmitter, and K. M. Leung, Phys. Rev. Lett. 67, 2295 (1991).

${ }^{8}$ W. M. Robertson, G. Arjavalingam, R. D. Meade, K. D.
Brommer, A. M. Rappe, and J. D. Joannopoulos, Phys. Rev. Lett. 68, 2023 (1992).

${ }^{9}$ S. L. McCall, P. M. Platzman, R. Dalichaouch, D. Smith, and S. Schultz, Phys. Rev. Lett. 67, 2017 (1991).

${ }^{10}$ R. D. Meade, K. D. Brommer, A. M. Rappe, and J. D. Joannopoulos, Appl. Phys. Lett. 61, 495 (1992).

${ }^{11}$ H. S. Sozuer and J. W. Haus, J. Opt. Soc. Am. B 10, 296 (1993).

${ }^{12}$ K. M. Ho, C. T. Chan, C. M. Soukoulis, R. Biswas, and M. Sigalas, Solid State Commun. 89, 413 (1994).

${ }^{13}$ E. Özbay, E. Michel, G. Tuttle, M. Sigalas, R. Biswas, and K. M. Ho, Appl. Phys. Lett. 64, 2059 (1994).

${ }^{14}$ E. Özbay, E. Michel, G. Tuttle, R. Biswas, K. M. Ho, J. Bostak, and D. M. Bloom, Opt. Lett. (to be published). 


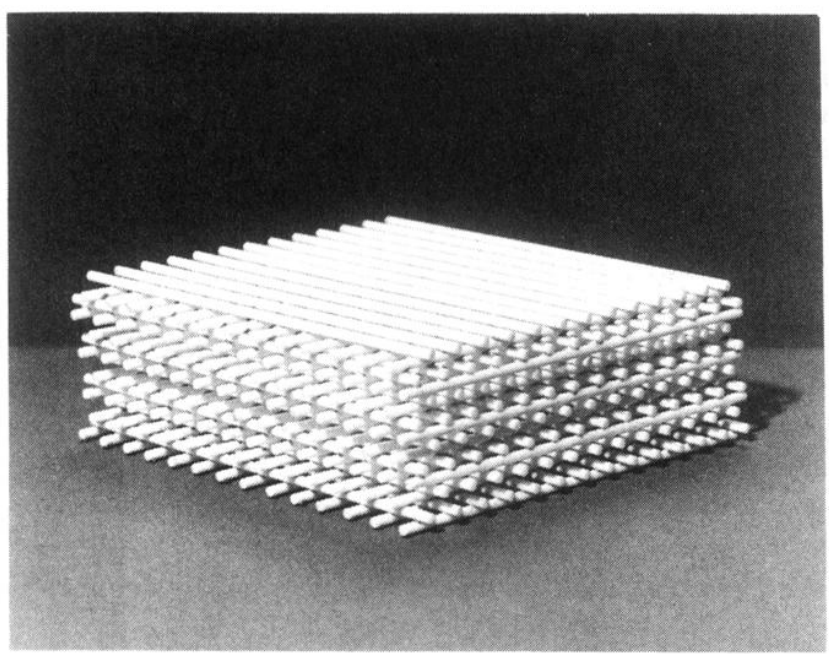

FIG. 1. Photograph of the three-dimensional photonic-gap structure. The crystal is built by stacking 16 layers of alumina rods (13 or 14 alumina rods on each layer) corresponding to four unit cells in the stacking direction. 